\title{
Mutations in the Drosophila melanogaster gene three rows permit aspects of mitosis to continue in the absence of chromatid segregation
}

\author{
Alastair Valentine Philp, J. Myles Axton*, Robert D. C. Saunders and David M. Glover† \\ Cancer Research Campaign Cell Cycle Genetics Group, Department of Anatomy \& Physiology, Medical Sciences Institute, \\ University of Dundee, Dundee DD1 4HN, Scotland, UK \\ *Present address: Whitehead Institute, Massachusetts Institute of Technology, Nine Cambridge Center, Cambridge, MA 02142, USA \\ †Author for correspondence
}

\section{SUMMARY}

We have cloned the three rows (thr) gene, by a combination of chromosome microdissection and $P$ element tagging. We describe phenotypes of embryos homozygous for mutations at the thr locus. Maternal mRNA and protein appear to be sufficient to allow 14 rounds of mitosis in embryos homozygous for $t h r$ mutations. However, a small percentage of cells in syncytial blastoderm stage $t h r$ embryos sink into the interior of the embryo as if they have failed to divide properly. Following cellularisation all cells complete mitosis 14 normally. All cells become delayed at mitosis 15 with their chromosomes remaining aligned on the spindle in a

\section{INTRODUCTION}

Thirteen cycles of rapidly alternating DNA synthesis and nuclear division follow the fertilisation of a Drosophila melanogaster egg. These cycles are unusual in at least two ways. Firstly, the syncytial division cycles lack the checkpoint that prevents entry into mitosis in the presence of unreplicated DNA. Thus injection of aphidicolin into embryos prevents DNA replication but has little effect on mitosis, with both centrosome replication and nuclear envelope breakdown cycles proceeding normally (Raff and Glover, 1988). Secondly, the mitotic A- and B-type cyclins do not undergo complete degradation at the metaphaseanaphase transition in the rapid division cycles (Maldonado-Codina and Glover, 1992).

After nuclear division 7 the majority of nuclei begin to migrate towards the periphery of the embryo, although some remain in the interior, cease dividing and become the polyploid yolk nuclei. The dividing nuclei have approached the surface by the time of the tenth nuclear division and form a monolayer just beneath it. During migration and in the syncytial blastoderm stages, when the nuclei are at the cortex, the time taken for each cell cycle increases, from 9 minutes for cycle 8 to 21 minutes for cycle 13 (Foe and Alberts, 1983). If any of divisions 11 to 13 at the embryo periphery are aberrant the affected nuclei may sink towards metaphase-like configuration, even though both cyclins $A$ and $B$ have both been degraded. As cyclin B degradation occurs at the metaphase-anaphase transition, subsequent to the microtubule integrity checkpoint, the delay induced by mutations at the thr locus defines a later point in mitotic progression. Chromosomes in the cells of $t h r$ embryos do not undertake anaphase separation, but remain at the metaphase plate. Subsequently they decondense. A subset of nuclei go on to replicate their DNA but there is no further mitotic division.

Key words: metaphase delay, mitosis, Drosophila, three rows the central part of the embryo (Sullivan et al., 1990). This loss of nuclei from the surface monolayer appears to be a generalised reaction to defective chromosome segregation (Minden et al., 1989; Warn et al., 1987). A small subset of nuclei become cellularised immediately upon reaching the posterior pole to form the pole cells, precursors of the adult germline. These cells no longer divide synchronously with the syncytial nuclei. Cellularisation of the remaining nuclei takes place in the interphase of cycle 14 following four further divisions at the cortex.

Maternally provided gene products are sufficient to drive the syncytial cycles (Szabad and Bryant, 1982). Mutations in these maternally expressed genes can give rise to mitotic defects in early embryos. Some of these genes, for example giant nuclei (gnu) (Freeman et al., 1986; Freeman and Glover, 1987), encode proteins that are apparently only required in the syncytial embryo. Females homozygous for mutations at the gnu locus produce eggs that undergo multiple rounds of DNA replication without any nuclear division. Centrosome replication occurs independently of the formation of these giant nuclei (Freeman et al., 1986; Freeman and Glover, 1987). Maternal effect mutations in other genes, plutonium and pan gu, have also been described to have similar phenotypes (Shamanski and Orr-Weaver, 1991). The autonomous replication of centrosomes appears to be a general consequence of disrupting mitosis in syn- 
cytial embryos and may be seen, for example, with combinations of mutant alleles of abnormal spindle (asp) that show a maternal effect mitotic phenotype (González et al., 1990).

For many of the gene functions necessary for mitosis the maternally provided mRNAs and proteins last until the third larval instar (Gatti and Baker, 1989). Thus a zygote homozygous for a mutation in certain genes essential for mitosis can survive until this late stage using the wild-type product supplied by its heterozygous mother. This perdurance of maternal protein has been clearly demonstrated for the asp product (Carmena et al., 1991), and it is inferred from the zygotic phenotype of other homozygous mutants lacking protein phosphatase PP1 87B (Axton et al., 1990), or a myosin light chain, spaghetti-squash (Karess et al., 1991), amongst other examples.

Other maternally supplied gene products only last until the time of cellularisation or shortly thereafter. The preeminent example is the product of the gene string (stg), a member of the $c d c 25$ family of mitotic inducers (Edgar and O'Farrell, 1989). Maternal transcripts of this gene become degraded at the time of cellularisation and this introduces an extended $\mathrm{G}_{2}$ phase into the later cell division cycles. Groups of cells (mitotic domains) enter mitosis 14 in a characteristic pattern (Foe, 1989) anticipated by the spatially and temporally regulated zygotic transcription of stg some 20 to 25 minutes earlier. In contrast to the syncytial divisions, the mitotic cyclins $\mathrm{A}$ and $\mathrm{B}$ now show distinct degradation at the metaphase-anaphase transition in the cells of the mitotic domains (Whitfield et al., 1990; Lehner and O'Farrell, 1989, 1990). There appears to be persistence of sufficiently high levels of maternal cyclin $A$ transcripts to permit mitoses 14 and 15 in the absence of zygotic expression. Embryos mutant for zygotic expression of cyclin $A$ arrest in the interphase before mitosis 16 (Lehner and O'Farrell, 1990). Embryos in which both cyclin $A$ and cyclin $B$ gene activities have been removed arrest during the interphase preceding mitosis 15 . This synthetic defect indicates some synergy of function between these two activities (Knoblich and Lehner, 1993).

While stg mutants are unable to enter mitosis 14 they are capable of considerable further development. However, the reduced number of cells means that although they differentiate segmental cuticular structures these are missing pattern elements (Hartenstein and Posakony, 1990). Work on the partial rescue of stg mutants, using a heat-inducible stg transgene, shows that there is a close correlation between the number of post-blastoderm mitoses completed and the number of rows of epidermal denticles that subsequently differentiate (Edgar and O'Farrell, 1990). The embryonic zygotic lethal mutation three rows (thr) was originally isolated because of its cuticular phenotype, a reduction in the number of rows of epidermal denticles (Nusslein-Volhard et al., 1984). Consideration of the arguments outlined above for string led to the idea that the cuticle phenotype in thr mutants could be explained by mitotic defects (Tearle and Nusslein-Volhard, 1988). In this paper we demonstrate that abnormal mitosis does indeed occur in thr embryos, and we characterise some aspects of the mitotic defects.

\section{MATERIALS AND METHODS}

\section{Molecular cloning}

In order to clone the $t h r$ gene, DNA from $t h r^{B H 9.8 / C y O}$ flies was digested with $\mathrm{XbaI}$ and ligated into Dash II (Stratagene), which was plated on the selective host Escherichia coli Q359 (Frischauf et al., 1983) to produce a library. DNA was obtained from polytene band 54F by microdissection of salivary gland chromosomes from wild-type $D$. simulans (a $D$. melanogaster sibling species) and amplified by PCR (Saunders et al., 1989). Microdissection was performed on $D$. simulans chromosomes in order to minimise problems associated with transposable elements within the dissected chromosome region (Sidén-Kiamos et al., 1990). The microcloned DNA hybridised exclusively to 54F-55A in situ. Plaques from the plated library were transferred onto duplicate filters for separate hybridisation with probes derived from the P element (p $\pi 25.1)$ and the PCR amplified microdissected DNA. Only clones hybridising to both probes were purified. These contain an insert comprising $18 \mathrm{~kb}$ of Drosophila DNA and a $2.9 \mathrm{~kb}$ P element. One such clone, 81.2 (Fig. 1), was used for further studies. A $5.2 \mathrm{~kb}$ EcoRI fragment from this phage was used to probe the Lorist 6 cosmid library of wild-type DNA, described by SidénKiamos et al. (1990). Six distinct clones were obtained from this screen, one of which is $15 \mathrm{C} 2$ (Fig. 1), which contains some 35 kb of Drosophila DNA that hybridises in situ to 54F3-6 on chromosome arm 2R. Altogether 50,000 plasmid clones from a 0-4 h embryonic cDNA library (Brown and Kafatos, 1988) were screened with a mixture of the $3.4 \mathrm{~kb}$ HindIII fragment and the $1.4 \mathrm{~kb}$ and $1.05 \mathrm{~kb}$ EcoRI-SacI restriction fragments from $15 \mathrm{C} 2$, which lie in the vicinity of the P element insertion site. One cDNA clone with an insert size of approximately $2.5 \mathrm{~kb}$ (2B1) and two smaller clones $(2 \mathrm{~A} 1,3 \mathrm{~A} 1)$ were recovered. All of these cDNA clones are incomplete, lacking sequence at the $5^{\prime}$ end.

\section{thr alleles}

The EMS (ethylmethanesulphonate)-induced $t h r^{1 B}$ allele and the $\mathrm{P}$ element-induced $t h r^{9.8}$ allele were used in the characterisation of the phenotype and the $t h r^{1 B}$ and $t h r^{S J B 22}$ alleles (both EMSinduced) were used in the rescue transformation experiments.

\section{Germline transformation and rescue}

This was performed essentially as described by Axton et al. (1990). Two constructs were separately microinjected into $w ; \Delta 2$ $3 \mathrm{Sb}$ e/TM2 embryos (Robertson et al., 1988). The larger, N15, is a $15 \mathrm{~kb}$ NotI fragment of cosmid $15 \mathrm{C} 2$ in the pCas 4 vector; 10 transformed lines were recovered from 49 fertile $\mathrm{G}_{0}$ adults $(20 \%$ transformants). The smaller construct, $\mathrm{RX} 2$, is a $9 \mathrm{~kb}$ fragment excised from cosmid $15 \mathrm{C} 2$ with $\mathrm{Xba \textrm {I }}$ and EcoRI partial digestion and ligated into the pw8 vector (Klemenz et al., 1987); 8 transformed lines were recovered from 62 fertile $\mathrm{G}_{0}$ adults (13\% transformants). The ability of the RX2 construct to rescue the lethality of thr mutations was tested using a line in which the transforming transposon was carried on the $\mathrm{X}$ chromosome. Females from such a line ( $\left.w^{a} \mathrm{P}\left[w^{+}, \mathrm{RX} 2\right] ; \mathrm{SM} 1 s p /+\right)$ were crossed to $w^{1118} / \mathrm{Y}$; thr $s p / C y O$ males. The $w^{+} ; s p \mathrm{~F}_{1}$ progeny were crossed inter se. The presence of $w^{+} ; C y^{+} s p^{-}$flies in the $\mathrm{F}_{2}$ progeny indicates that the RX2 construct can rescue lethality of $t h r^{1 B}$ mutations. A similar approach, but using $C y O d p$ in place of $S M 1 s p$, was used to show that RX2 will rescue flies homozygous for thr $r^{S J B 22}$.

\section{Sequence analysis}

cDNA 2B1 was cleaved into two EcoRI fragments of 1.7 and 1.1 $\mathrm{kb}$ and these were each ligated into both of the pBluescript ${ }^{\circledR} \mathrm{KS}+$ and pBluescript ${ }^{\circledR} \mathrm{SK}+$ vectors (Stratagene). These constructs were oriented so that the sequencing primer (M13-20) was closest to 
the $5^{\prime}$ ends of both fragments in pKS + and to the $3^{\prime}$ ends in $\mathrm{pSK}+$. Series of nested exonuclease III deletion subclones were prepared, starting from the $X b a \mathrm{I}$ site of $\mathrm{pKS}+\left(5^{\prime}\right.$ to $\left.3^{\prime}\right)$ and from the XhoI site of the pSK+ (3' to $\left.5^{\prime}\right)$, by the method of Henikoff (1987). Double-stranded plasmid deletion subclones were packaged as single-stranded phage by superinfection with M13 MK07 helper phage and prepared for dideoxynucleotide sequencing by standard procedures (Sambrook et al., 1989); 0.5 and $1.7 \mathrm{~kb}$ SalI/HindIII cDNA fragments were also sequenced. We were unable to isolate a full-length cDNA from available libraries and so determined the sequence of the $5^{\prime}$ end of the gene from genomic DNA. The appropriate 2.4, 1.6 and $4 \mathrm{~kb}$ EcoRI genomic fragments were cloned into $\mathrm{pKS}+$ and $\mathrm{pSK}+$, and deletion series were constructed starting from the SacII site of KS and the SalI site of SK; $2.3 \mathrm{~kb}$ SpeI/ EcoRI and 560 bp PstI/HindIII fragments of genomic DNA were also sequenced. In order to positively identify the $5^{\prime}$ end of the thr mRNA we used nested PCR primers, single-stranded ligation

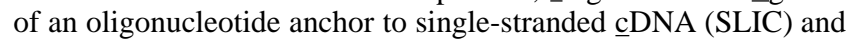
rapid amplification of $\underline{\mathrm{c} D N A}$ ends (RACE) PCR to obtain a cDNA fragment corresponding to this region of the message (Clonstech 5' AmpliFINDER ${ }^{\text {TM }}$ RACE kit). The resulting PCR product was sequenced using ${ }^{32} \mathrm{P}$-end-labelled specific sequencing primers and the BRL dsDNA cycle sequencing system. Accumulated sequence was assembled using the Microgenie software.

\section{Immunostaining of embryos}

Embryos were collected, dechorionated and fixed in paraformaldehyde as described previously (Maldonado-Codina and Glover, 1992) with the following modifications. A balancer chromosome CyO ftz-lacZ (kind gift from Sarah Bray) was used to distinguish thr $r^{+}$embryos from their homozygous mutant siblings. Taxol was only used when microtubule distribution was to be examined and instead of immediately devitellinising embryos following fixation they were washed overnight in $0.1 \%$ Triton $\mathrm{X}-100$ in PBS (PBTrX). Embryos were then stained for $\beta$-galactosidase activity by incubation in $0.28 \% \mathrm{X}$-gal in $\mathrm{Fe} / \mathrm{NaP}$ buffer $(\mathrm{pH} 7.2,7.2 \mathrm{mM}$ $\mathrm{Na}_{2} \mathrm{HPO}_{4}, 2.8 \mathrm{mM} \mathrm{NaH} \mathrm{PO}_{4}, 3.05 \mathrm{mM} \mathrm{K} 3 \mathrm{Fe}(\mathrm{CN})_{5}, 3.05 \mathrm{mM}$ $\mathrm{K}_{4} \mathrm{Fe}(\mathrm{CN})_{6}, 150 \mathrm{mM} \mathrm{NaCl}$ and $\left.50 \mathrm{mM} \mathrm{MgCl}_{2}\right)$ and washed twice in PBTrX. Methanol devitellinisation, antibody staining and mounting were as previously described (Whitfield et al., 1990). Images were captured using a Bio-Rad MRC 600 laser-scanningconfocal head, in conjunction with a Nikon Optiphot microscope and assigned the false colours indicated in the figure legends. Colour prints were obtained using a Sony Color video printer UP5000-P.

\section{Antibodies}

The polyclonal rabbit serum Rb271 (against cyclin B) (Whitfield et al., 1990) was used at a dilution of 1 in 500. The rat monoclonal YL1/2 (Seralabs) directed against tyrosinated tubulin was used at 1 in 10 (hybridoma supernatant) and 1 in 100 (ascites).

\section{RESULTS}

\section{The identification of the thr gene}

The embryonic lethality resulting from thr mutations is uncovered by the deficiencies $D f(2 R) P c l 7 B$ and $D f(2 R) P c l 11 B$ but not by $D f(2 R) P c l W 5$, thus placing $t h r$ on the right arm of chromosome 2 between 54F and 55A1 (Fig. 1A). We examined $t h r$ alleles generated by J. P. Gergen using P-M hybrid dysgenesis to determine whether a P element transposon was inserted at this chromosomal site. The P element probe p $\pi 25.1$ (O'Hare and Rubin, 1983) hybridized in situ to an element located at 54F3-6, which was present in stocks $t h r^{B H 9.6}$ and $t h r^{B H 9.8}$ but not in the wild-type dysgenic revertant $t h r^{B H 9.9}$. This offered the possibility of cloning the tagged gene. We therefore constructed a library of recombinant bacteriophage using DNA from flies carrying the $t h r^{B H 9.8}$ mutation, maintained over the $\mathrm{CyO}$ balancer chromosome, which we screened using both a $\mathrm{P}$ element probe and a probe prepared from DNA microdissected from band $54 \mathrm{~F}$ of wild-type $D$. simulans salivary gland chromosomes and amplified by PCR (see Materials and Methods). DNA from one such double-pos-

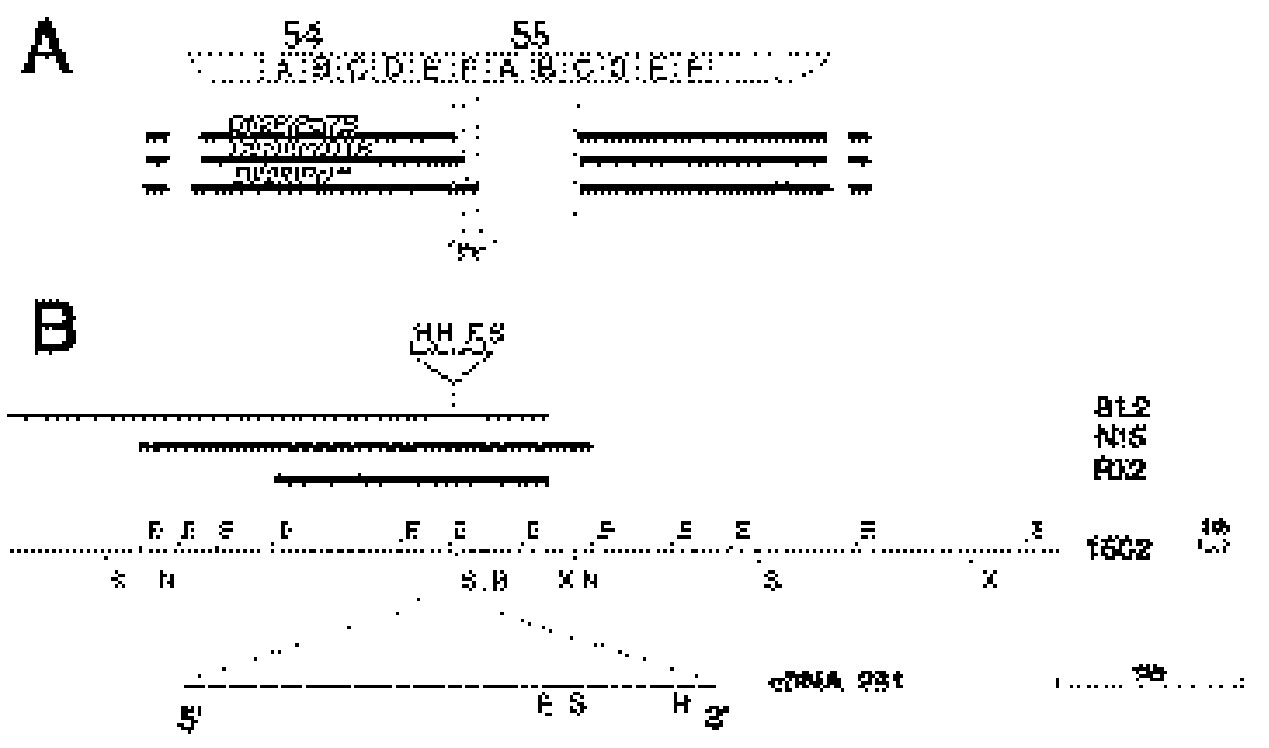

transformation. The embryonic cDNA $2 \mathrm{~B} 1$ is shown below on an enlarged scale, the broken lines indicating its relationship to the equivalent region of genomic DNA. E, EcoRI; B, BamHI; H, HindIII (only sites for P element and cDNA shown); N, NotI; S, SalI; X, XbaI.
Fig. 1. Cytogenetic and molecular maps of thr. (A) Diagrammatic representation of salivary gland divisions 54 and 55 to show regions uncovered by three chromosomal deficiencies. $t h r$ mutations are uncovered by the deficiencies $D f(2 R) P c l 7 B$ and $D f(2 R) P c l 11 B$ but not by $D f(2 R) P c l W 5$. (B) Map of the $t h r$ locus. The extent of lambda clone 81.2 , bearing the $\mathrm{P}$ element from chromosomal region $54 \mathrm{~F}$ of the $t h r^{B H 9.8}$ chromosome is shown above the restriction map cosmid $15 \mathrm{C} 2$ containing the corresponding wild-type DNA. $\mathrm{N} 15$ and $\mathrm{RX} 2$ are restriction fragments of $15 \mathrm{C} 2$ that restore $t h r^{+}$function when reintroduced to flies by germline 
-800 atcaataagcaaaaaattgtatcgttatcggaaatgagcatggaagtttagtattttccacaagtatttgtgttcagatatgaaaactagaaatatcga

-700 tgttatcgttaaaggatttccagctttagcatggacggtcacactggatctcaaaatctggcgcaaagcaacaaaaaaggaagcgtcgcagttaaact

-600 agttaaatgtgccaattcccttgaaaaatgtctactgatatagccacccagctgaagggcagccgctccgatgtggaaaatgtccgcaaaacggtgg

-500 aagccaaattccgggagttgtctggcgatggactacctctgagatacgaagtgaatgtattgcgccatatttgcctcgccttaaaggataatctgcacc

-400 agaactcggatctttactgcgacatcatgggcataatgctgccacgcgtagttccctgcgaggagaagccaagcttatgggaagcgcacttgtccagcc

-300 tgcggtacatacatcatggcttgtttcatcaggtaggagtcgctataagaatcttcgaatcttctaaccaattctcccgttcacagcgatccattgaa

-200 gcctgtcagaagcttacaacctcatccgacaacaaccctgccgtctgcaagaggaatcagactacaaatatatctggacatacatt

-100 aatggtttccatgtgctgctgcagaagcaaaactccccttggaagctacaagtcaattgtgttatgctttggaatcattgggagatctattcgcagcc

1 Atg ACG CAA AGg CAA AtA Agt CTA TGC GCA ACG CTT CTA GTt CAA CTA AAC GAg Agt tTA TTt GGC AAA AgA Agt $\begin{array}{lllllllllllllllllllllllllllllllllll}1 & M & T & Q & R & Q & I & S & L & C & A & T & L & V & Q & L & N & E & S & L & F & G & K & R & S & S\end{array}$

76 AgA TCA TTC TTC AAg TCC CTA AGC TTC CTG CCG TCG GAg Agt CTT GCC AAA ATg tTC AAC CCG CTG CTT ATG CTC

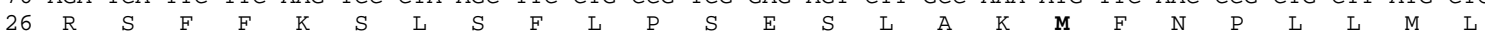

151 CTG GCC AGC AGC ACC AGT TCG AAC CTG GCC AAC TTA TTC CCT GAg tgC CTG Agt CTC ACG TtG GCT CTC GTA CAA $\begin{array}{lllllllllllllllllllllllllllllllllllll}51 & \text { L } & \text { A } & \text { S } & \text { S } & \text { T } & \text { S } & \text { S } & \text { N } & \text { L } & \text { A } & \text { N } & \text { L } & \text { F } & \text { P } & \text { E } & \text { C } & \text { L } & \text { S } & \text { L } & \text { T } & \text { L } & \text { A } & \text { L } & \text { V } & Q\end{array}$

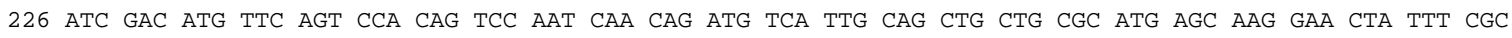
$\begin{array}{lllllllllllllllllllllllllllllllllll} & \mathrm{I} & \mathrm{D} & \mathrm{M} & \mathrm{F} & \mathrm{S} & \mathrm{P} & \mathrm{Q} & \mathrm{S} & \mathrm{N} & \mathrm{Q} & \mathrm{Q} & \mathrm{M} & \mathrm{S} & \mathrm{L} & \mathrm{Q} & \mathrm{L} & \mathrm{L} & \mathrm{R} & \mathrm{M} & \mathrm{S} & \mathrm{K} & \mathrm{E} & \mathrm{L} & \mathrm{F} & \mathrm{R}\end{array}$

301 CAG GAA TCA AAC CTG TGC TAT GCT CTA CAG CTG ATG TAC TAC TAT ATC AAg tTA ATC TTt GTT CGG GAA CCA ACA

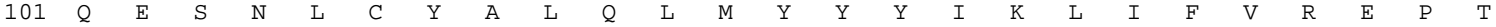
376 GGC GAC TTT AAg CGC ACC TAC ATt GAC TTG TCT AGC AAg TTt CAG CAC TTC TTC GAG CAC AAA GTA GCC TCG CAT $\begin{array}{lllllllllllllllllllllllllll}126 & G & D & F & K & R & T & Y & I & D & L & S & S & K & F & Q & H & F & F & E & H & K & V & A & S & H\end{array}$

451 GCC AAA GAA CAG TGG CTA GCG GAT TTT CTG GTG GCC ATT CAA TTG CTA CAG GTG CTC ATC CAT CAA AGT AAC AGT

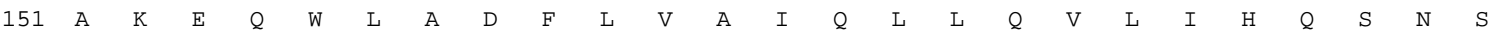

526 AAg TTG CAg AGT CCT TTT CAg ATA TTT TGg CAg CAg TTT GAC GGA GAg AgC AgT CCC GAg ATC TAC ACA GCG CAC

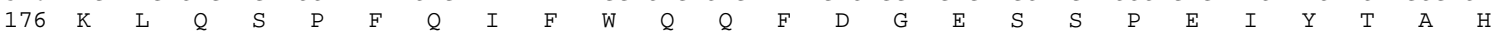

601 TTT CAG TTG CTT CAG ACG TGT GCT AGC TTG GCG GTT AAT ATT ACG AgG AgT CCT TTG GGC TGC AGT TGC TCC CAC

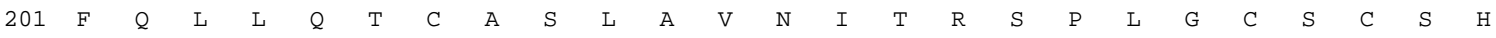

676 GAG GCA TGC AAg AGC GTG CGA CGG CAC TGC ATA TTG GCG TAT GGA TtG TGC GCA TTA GAT GCG TAT ATt AAt TGG

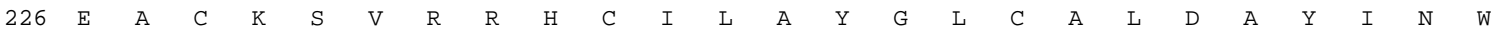

751 AAA CCG GCG GCG GAG CAG AGA GCA AAT GTG gtgggtatttttatctaatttgtggaatttaatgctacatttcaatactattgcag $\begin{array}{llllllllllllllllll}251 & \text { K } & \text { P } & \text { A } & \text { A } & \text { E } & \text { Q } & \text { R } & \text { A } & \text { N } & \text { V }\end{array}$

839 AGC CCT CAC AAg CCC TTG CTG GGA GTA GTC AAA TAC TCA ATG GAT GTG GCT AAG ACC ATG AAg TGC TTG GGT CCC $\begin{array}{llllllllllllllllllllllllllllllllll}261 & \mathrm{~S} & \mathrm{P} & \mathrm{H} & \mathrm{K} & \mathrm{P} & \mathrm{L} & \mathrm{L} & \mathrm{G} & \mathrm{V} & \mathrm{V} & \mathrm{K} & \mathrm{Y} & \mathrm{S} & \mathrm{M} & \mathrm{D} & \mathrm{V} & \mathrm{A} & \mathrm{K} & \mathrm{T} & \mathrm{M} & \mathrm{K} & \mathrm{C} & \mathrm{L} & \mathrm{G} & \mathrm{P}\end{array}$

914 ACC AGT GTG GAg ATC ATT AAg CTA GTG CGC CAg CTG ACA TAC GTg GCT GAT CAG GTC ACC TGT CCG GAG CAA ATG $\begin{array}{llllllllllllllllllllllllllllllllllllllllll}286 & \mathrm{~T} & \mathrm{~S} & \mathrm{~V} & \mathrm{E} & \mathrm{I} & \mathrm{I} & \mathrm{K} & \mathrm{L} & \mathrm{V} & \mathrm{R} & \mathrm{Q} & \mathrm{L} & \mathrm{T} & \mathrm{Y} & \mathrm{V} & \mathrm{A} & \mathrm{D} & \mathrm{Q} & \mathrm{V} & \mathrm{T} & \mathrm{C} & \mathrm{P} & \mathrm{E} & \mathrm{Q} & \mathrm{M}\end{array}$

989 TCC GTG CTG CTG CCA CTT TTG GAG CCA CTG CAG AAG CTG CGA CCT TTG GTT GCC GAC CAG GAT ATG AGC AGt TTA

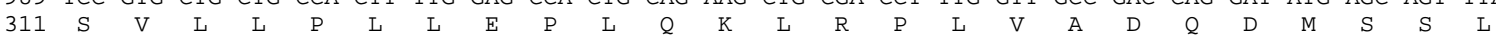

1064 CTC CGA CGC CTC TTT AAG GCC AGC TCC CAT TGC GGC GAT TCC AAT ATA GCT TGT CGA ATt CAA GCT AGT TAT TTG $\begin{array}{llllllllllllllllllllllllll}336 & \mathrm{~L} & \mathrm{R} & \mathrm{R} & \mathrm{L} & \mathrm{F} & \mathrm{K} & \mathrm{A} & \mathrm{S} & \mathrm{S} & \mathrm{H} & \mathrm{C} & \mathrm{G} & \mathrm{D} & \mathrm{S} & \mathrm{N} & \mathrm{I} & \mathrm{A} & \mathrm{C} & \mathrm{R} & \mathrm{I} & \mathrm{Q} & \mathrm{A} & \mathrm{S} & \mathrm{Y} & \mathrm{L}\end{array}$

1139 GCC TCG ATT ACG AAT CCG GCA CGA TTA AGA TCA CAG GTC TGT TTG TAC TAT CAC AAT CTG GGA AAA AAG GGC ACC

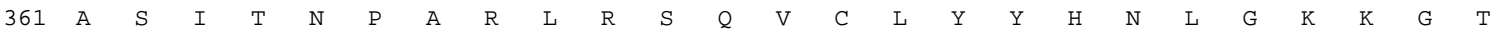

1214 GAg ATC AAA AGg TGT GTC TAC GAg TGG CAC GAg TCC ACG CCA CTA CCT TTt CCT CTC ACT CCG GAC CAG AAg AAA $\begin{array}{llllllllllllllllllllllllllllllllllllllll}386 & \mathrm{E} & \mathrm{I} & \mathrm{K} & \mathrm{R} & \mathrm{C} & \mathrm{V} & \mathrm{Y} & \mathrm{E} & \mathrm{W} & \mathrm{H} & \mathrm{E} & \mathrm{S} & \mathrm{T} & \mathrm{P} & \mathrm{L} & \mathrm{P} & \mathrm{F} & \mathrm{P} & \mathrm{L} & \mathrm{T} & \mathrm{P} & \mathrm{D} & \mathrm{Q} & \mathrm{K} & \mathrm{K}\end{array}$

1289 CAG CTG TAT GAT ACC GAT TTC TTT GCC TTA CTA CAC TAT TTG AGG AGT CCT TCT ACG GCT CAT ATG GAA TCA CTA

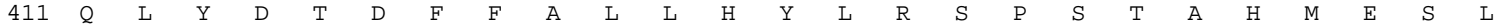

1364 ATt CGT TGC CGA ACG AGT GAC TAT CAT CTG GTA CTC TTG GCC AGA CAA ATg CGA AAC GAT GAC TCG*AtT TCG AAg $\begin{array}{llllllllllllllllllllllllllllllll}436 & \text { I } & \text { R } & \text { C } & \text { R } & \text { T } & \text { S } & \text { D } & \text { Y } & \text { H } & \text { L } & \text { V } & \text { L } & \text { L } & \text { A } & \text { R } & \text { Q } & \text { M } & \text { R } & \text { N } & \text { D } & \text { D } & \text { S } & \text { I } & \text { S } & \text { K }\end{array}$

1439 AAA TGC ATA GAG GTT CAT GAT AAG CTA AGG CAA CAA CGT TCG CTC AGT CGA ATG GAT AAC TTG TGC CTG GGC CAC

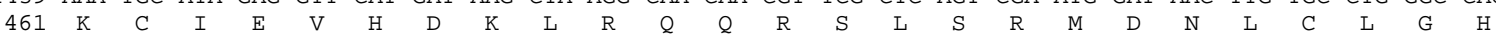

1514 GCA AGT GTG GGA CTA CTA CTG GAC GCA CTG GAG GCT CAA AAA ACC AAA GTT TCC ACC AAG GAG ATA ACG GAA AAT

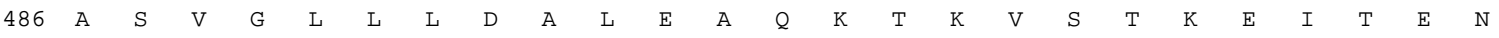

1589 Atg tTC GAG GAg CTG CTA CTC AGC AAg AAT tTA TGG CAg Atg AAC AtA CAA AgG GAg CAG CGA tTG GTC AAC tAT

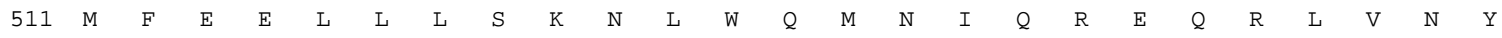

1664 GCT AGT GAA GCC ATC TCG GCC TTC AGC AAC TTC TTC GAT CGA GCA GAT CAA GAG CCA TTG AGC GCA AAT GAA ACG

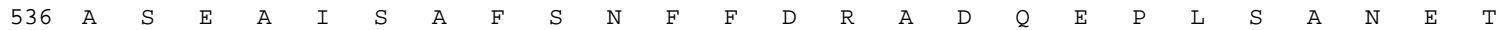

1739 TCT ATt GAT TGG GAg GCA TTG ATT GAC GAT GCC ATC GCT ACT GCC AAT GCA CTT TCA AGT ATG GGG TAT CAG TCA $\begin{array}{llllllllllllllllllllllllllllllllllllll}561 & \mathrm{~S} & \mathrm{I} & \mathrm{D} & \mathrm{W} & \mathrm{E} & \mathrm{A} & \mathrm{L} & \mathrm{I} & \mathrm{D} & \mathrm{D} & \mathrm{A} & \mathrm{I} & \mathrm{A} & \mathrm{T} & \mathrm{A} & \mathrm{N} & \mathrm{A} & \mathrm{L} & \mathrm{S} & \mathrm{S} & \mathrm{M} & \mathrm{G} & \mathrm{Y} & \mathrm{Q} & \mathrm{S}\end{array}$ 1814 GAA GAg GAT GAT GCC TGG CTG TTG CTT CTG AGg ATg GGT CGC TTG CTG GAA GAT CGT TTT ACC TAT CTG CGT GCC

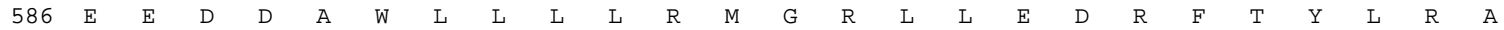




\footnotetext{
1889 CTA AAT CAT TTT CTG TCA CAG AAT GAG GTT AGT TCT CGT TTA AAT CTG AAA CTC GGC GAG GAA GTG GAA GTA GCA

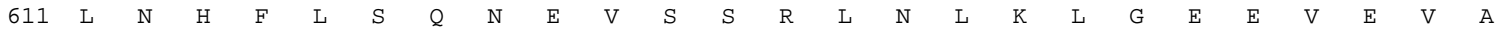
1964 GAg GAA TTG CTG GAT GAT TTG TGg CCT CAA TTG AAA AAT GgC AAA tTC tTC AAg CGT CAg CAA ACT ACG GTA ATG

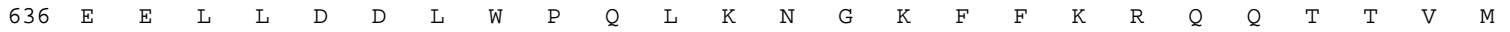
2039 CTC TGT TTT TGT CAC CTC GCC AGT TAC TAT GCC AGA ATg GAA TGC TAT Agt CAT GCC CAG TTG CTT CTA tTG CAT $\begin{array}{llllllllllllllllllllllllll}661 & \mathrm{~L} & \mathrm{C} & \mathrm{F} & \mathrm{C} & \mathrm{H} & \mathrm{L} & \mathrm{A} & \mathrm{S} & \mathrm{Y} & \mathrm{Y} & \mathrm{A} & \mathrm{R} & \mathrm{M} & \mathrm{E} & \mathrm{C} & \mathrm{Y} & \mathrm{S} & \mathrm{H} & \mathrm{A} & \mathrm{Q} & \mathrm{L} & \mathrm{L} & \mathrm{L} & \mathrm{L} & \mathrm{H}\end{array}$ 2114 GTG GAA CAA CTT CGC GAA GAg TTT CCT GAg AGA CAA GGA AAA AgT GAT ATt GTA TTG CTT ACA CTG CAA ACG GTG

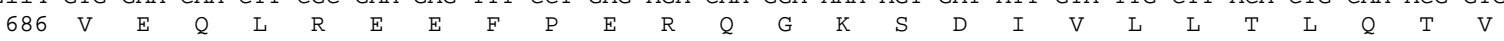
2189 CGC TTT CGA ATt GGg TAT CAg CAA AGg AAg CCA ACG AAT TGC AgG CTG CCG ACT CCT CTG CGT CAA TTG GAC ATt $\begin{array}{lllllllllllllllllllllllllllll}711 & R & F & R & I & G & Y & Q & Q & R & K & P & T & N & C & R & L & P & T & P & L & R & Q & L & D & I\end{array}$

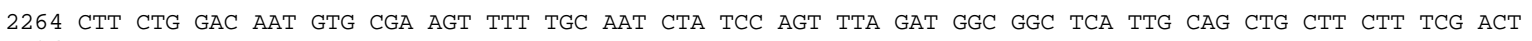

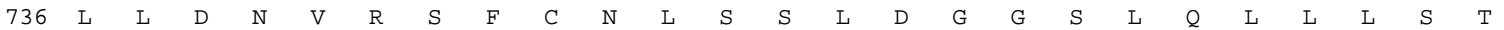
2339 CTT GTC AGg GAA AgC ACC GAg TGC TCT GCG AAC AGA TTA AgC GAA AGA CTG TCC TTC TCC AAC ATT GCA CTA CAT $\begin{array}{lllllllllllllllllllllllllllllllll} & \mathrm{L} & \mathrm{V} & \mathrm{R} & \mathrm{E} & \mathrm{S} & \mathrm{T} & \mathrm{E} & \mathrm{C} & \mathrm{S} & \mathrm{A} & \mathrm{N} & \mathrm{R} & \mathrm{L} & \mathrm{S} & \mathrm{E} & \mathrm{R} & \mathrm{L} & \mathrm{S} & \mathrm{F} & \mathrm{S} & \mathrm{N} & \mathrm{I} & \mathrm{A} & \mathrm{L} & \mathrm{H}\end{array}$

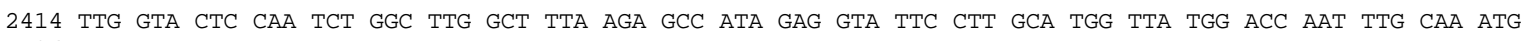

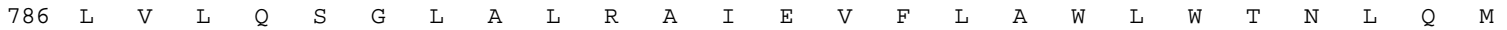
2489 GAA AGT TTC GAC AAg GCG CAA TCG AAG TTG AGA CTC ATC GAG CAT TGT TTA GGT ATA AAA CAG CTG AAT CCA ACG

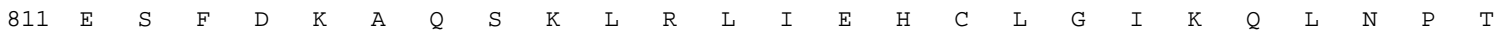
2564 Agt AgG CCG GAA AAg GAA GCG ATt AAg GAT GTA GCA ATT AgT GAT CTG GCT Agt AAT ATG CAT CTC CTC CAA TTG

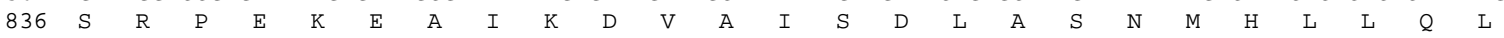
2639 GTG GAG CCG ATC AGg AAg CAg CAG CTA TTA AAT ATG GCC TCG CCG AAT CTG CTC AAA ATG CGA CCA CAT AGC CCA $\begin{array}{llllllllllllllllllllllllllll}861 & \mathrm{~V} & \mathrm{E} & \mathrm{P} & \mathrm{I} & \mathrm{R} & \mathrm{K} & \mathrm{Q} & \mathrm{Q} & \mathrm{L} & \mathrm{L} & \mathrm{N} & \mathrm{M} & \mathrm{A} & \mathrm{S} & \mathrm{P} & \mathrm{N} & \mathrm{L} & \mathrm{L} & \mathrm{K} & \mathrm{M} & \mathrm{R} & \mathrm{P} & \mathrm{H} & \mathrm{S} & \mathrm{P}\end{array}$

2714 AAT CCC CAA CTG GAC TTG GAT CGC TAC ATA ACA CTC GAT GTG GCG CCA GCG AAT CTT CGA GAG AAT TCC CAG CTG

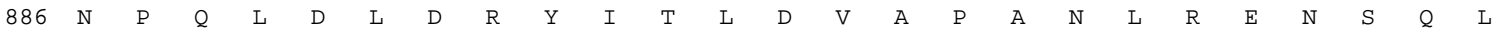
2789 CAA TGC TTG TAC TTt GTA ACG GGC TGT CtA CAT GCA CGT CTC CGC tTt CTG CAg AgA AAC AgC GAA CAA tTG GAG

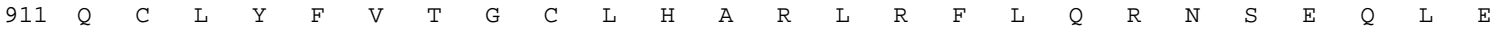

2864 GAg TTC TAT GGA AGA GCG CAT AAC TGG ATG CAg GAA AAA CCT CCG ATG AgT AgC GCT TTG TAT CCC ATG TTG CAT $\begin{array}{llllllllllllllllllllllllllllllll}936 & \text { E } & \text { F } & \text { Y } & \text { G } & \text { R } & \text { A } & \text { H } & \text { N } & \text { W } & \text { M } & \text { Q } & \text { E } & \text { K } & \text { P } & \text { P } & \text { M } & \text { S } & \text { S } & \text { A } & \text { L } & \text { Y } & \text { P } & \text { M } & \text { L } & \text { H }\end{array}$ 2939 GCC CAg CAg CTG TAT CAT CTC AAC TAT CTT CGC TTT GCG CGA AAg CAT GTA GAg GCT AtA TCA ACG GCT CAA TTG

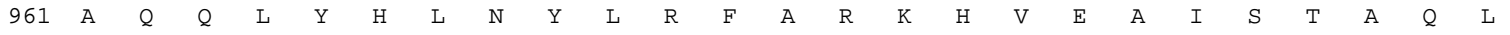
3014 GGC CTT AAA ATG CGA TCA CGC GCG GTC GAC AtT AAt tTC GAg TAC AAt tTt tTG GCT CAg CTA AAg ACG GCT CAg

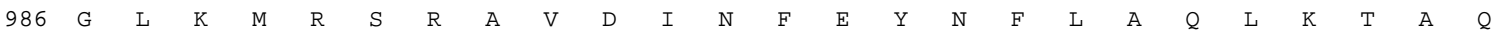
3089 TTA GAA CTC AAA CCC GTG GGC CAG GAT AAG CCA CAG GTC AAA ATC CTT CGA CGT GCT TTG GTA TTC AAT CAC TCA

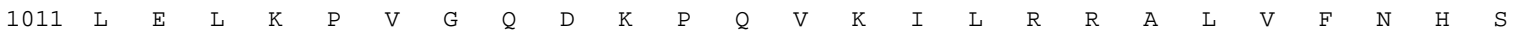
3164 CCC GAA GAT AAG AAA CGA ACC GCA ACA GGA TCG GTT TCA GCA GTC AAg AAT ACG GCG TCT AAA GTT AAA CAG TCG $\begin{array}{lllllllllllllllllllllllllll}1036 & P & E & D & K & K & R & T & A & T & G & S & V & S & A & V & K & N & T & A & S & K & V & K & Q & S\end{array}$ 3239 GCC AAA AAG GCA CCT CGA TTC AGA ATT TAC GAg GAG CTG GAA CTA CGA CCA CCA AGT GCT ACC AGT TGC AGT AGC

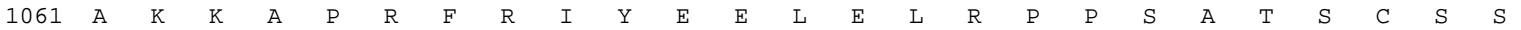
3314 AGC GGT GGC AGC GGA ACA GAg AAT ACA CCG CCT TCG GAT CAC GTG GAT CTA AAT GCC TGT CAA GCG ATC GAG ATA

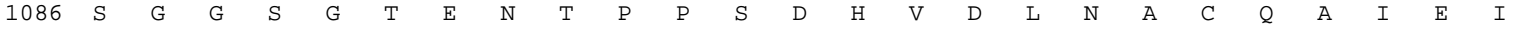
3389 AGC GAC GAT GAC GAT TCA CCT TTG GTG TCC ACA AAG AAG ACA CAA CCA AAA AGT AGG GAG AAG GCA AAA CCC AAG

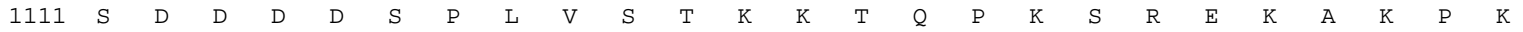
3464 GCC ACA TCC AAA GCT TGT AAA GTC CTA ACA TTG GAT AAT AGC TTG GAA ATA GTA GAA ACG CCG ACA ATA ACT ACA

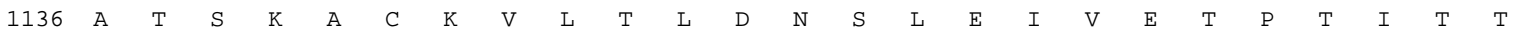
3539 AGT ACA CGG AGC ACC AGA GCC AGG CTG CGC AAC CAG TAG agacaccaaagacagcgactctttcatccaagcgaacgaggcgccag $\begin{array}{llllllllllllll}1161 & \mathrm{~S} & \mathrm{~T} & \mathrm{R} & \mathrm{S} & \mathrm{T} & \mathrm{R} & \mathrm{A} & \mathrm{R} & \mathrm{L} & \mathrm{R} & \mathrm{N} & \mathrm{Q} & \otimes\end{array}$

3625 gttttggaagcacaggctcccgaaacggagtccattagcacacgcacacggcatcggcactgattagatagtagtctccttagttcgtatttattctta 3725 tcgaaatgtcttggtatttaaatatatatatatattgtgtgt
}

Fig. 2. Sequence of the three rows transcription unit. Nucleotide and predicted amino acid sequence of thr. The first bold-type methionine residue following the in-frame stop (nucleotides -54 to -52) shows a 5/14 match to the published translation initiation consensus (Cavener and Ray, 1991). The second bold-type methionine shows a 8/14 match, maintaining all five of the most conserved (capitalised) residues in the consensus cacaaCAaaATGgc. Upper case letters represent residues conserved in more than 50\% of genes studied (Cavener and Ray, 1991) and lower case letters indicate the most frequent base at a position where no base occurs more than $50 \%$ of the time. * indicates a position (nucleotide 1429) where we read a $\mathrm{C}$ residue in a genomic clone and a $\mathrm{G}$ residue in a cDNA clone. A poly(A) tail follows the last indicated nucleotide. 
itive recombinant phage identified in this screen, 81.2 (Fig. 1B), carries a segment of Drosophila DNA bearing a 2.9 $\mathrm{kb} \mathrm{P}$ element and was used to screen a cosmid library of wild-type Drosophila DNA (Materials and Methods). One of the resulting clones, $15 \mathrm{C} 2$, hybridises to 54F3-6 in situ and contains $35 \mathrm{~kb}$ of DNA around the site of the $\mathrm{P}$ element insertion found in the $t h r^{B H 9.8}$ chromosome (Fig. 1B). A comparison of the restriction maps of the phage 81.2 , the cosmid 15C2 and an embryonic cDNA, 2B1, isolated using probes from the $\mathrm{P}$ element insertion site (Materials and Methods) indicated that the $\mathrm{P}$ element in $t h r^{B H 9.8}$ is inserted in a SalI-EcoRI fragment of approximately $200 \mathrm{bp}$, which is present in both genomic DNA and the cDNA. It is therefore likely that the $\mathrm{P}$ element disrupts the expression of an embryonic transcript.

In order to confirm that the thr gene lies at this site, we
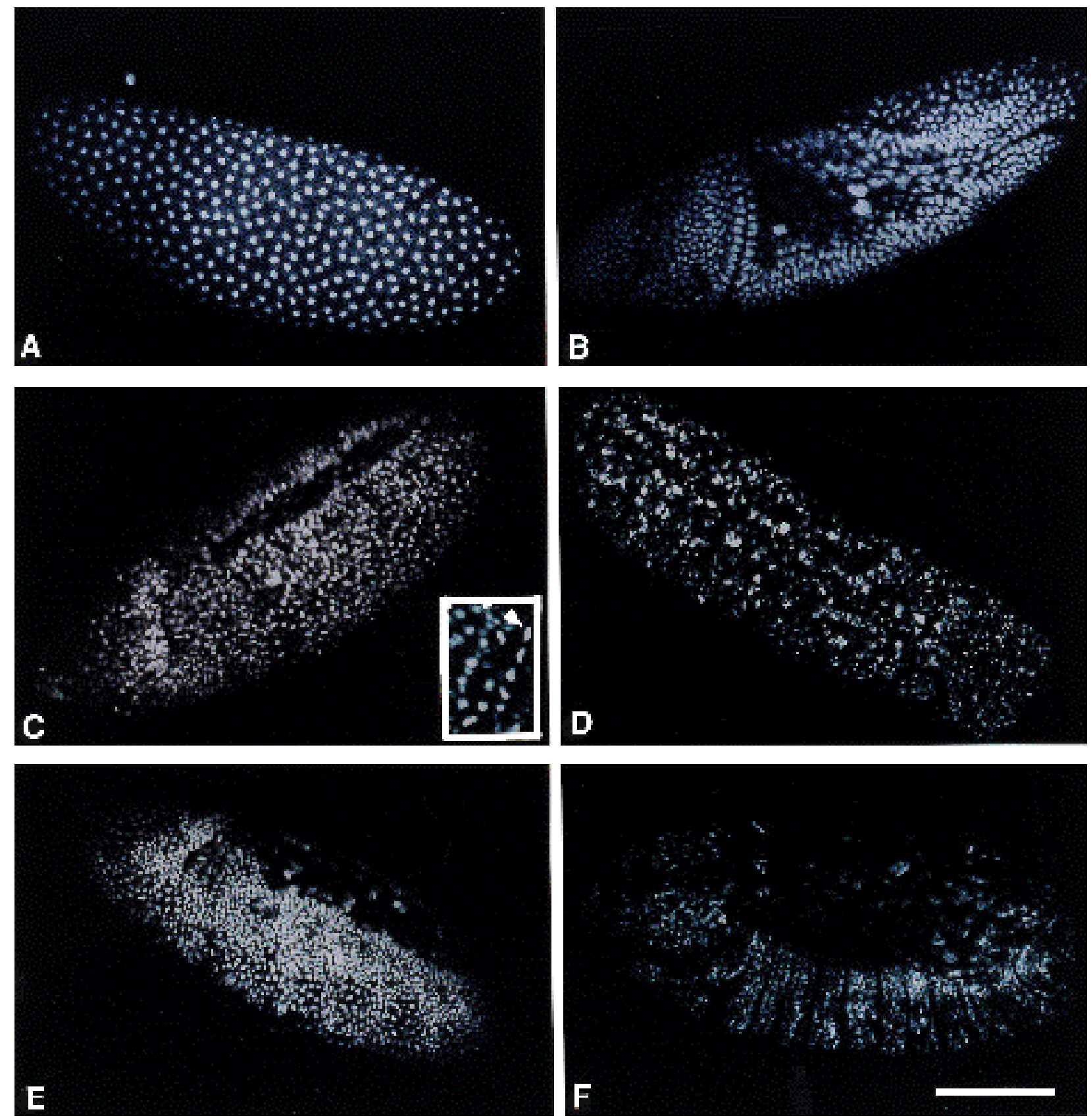

Fig. 3. Development of $t h r$ (A-D) and wild-type (E,F) embryos. Embryos have been fixed and stained with propidium iodide to reveal DNA as described in Materials and Methods. thr embryos in (A-C) are at the following division cycle stages: (A) cycle 12; (B) cycle 14; (C) cycle 15. The embryo in (C) shows mitotic domains that appear larger than usual as they have coalesced. The inset in (C) shows a twofold enlargement of one of these areas. Corresponding metaphase figures are indicated by arrowheads in the main panel and in the inset. No further cell division occurs following cycle 15, and embryos develop the appearance shown in (D) by $7 \mathrm{~h}$ AED. The wild-type embryo (approx. $5.5 \mathrm{~h}$ AED) in (E) is undertaking mitosis 15. A later embryo (approx. $7 \mathrm{~h} \mathrm{AED)} \mathrm{is} \mathrm{shown} \mathrm{in} \mathrm{(F)} \mathrm{following} \mathrm{germ-band}$ retraction. Bar, $100 \mu \mathrm{m}$. 


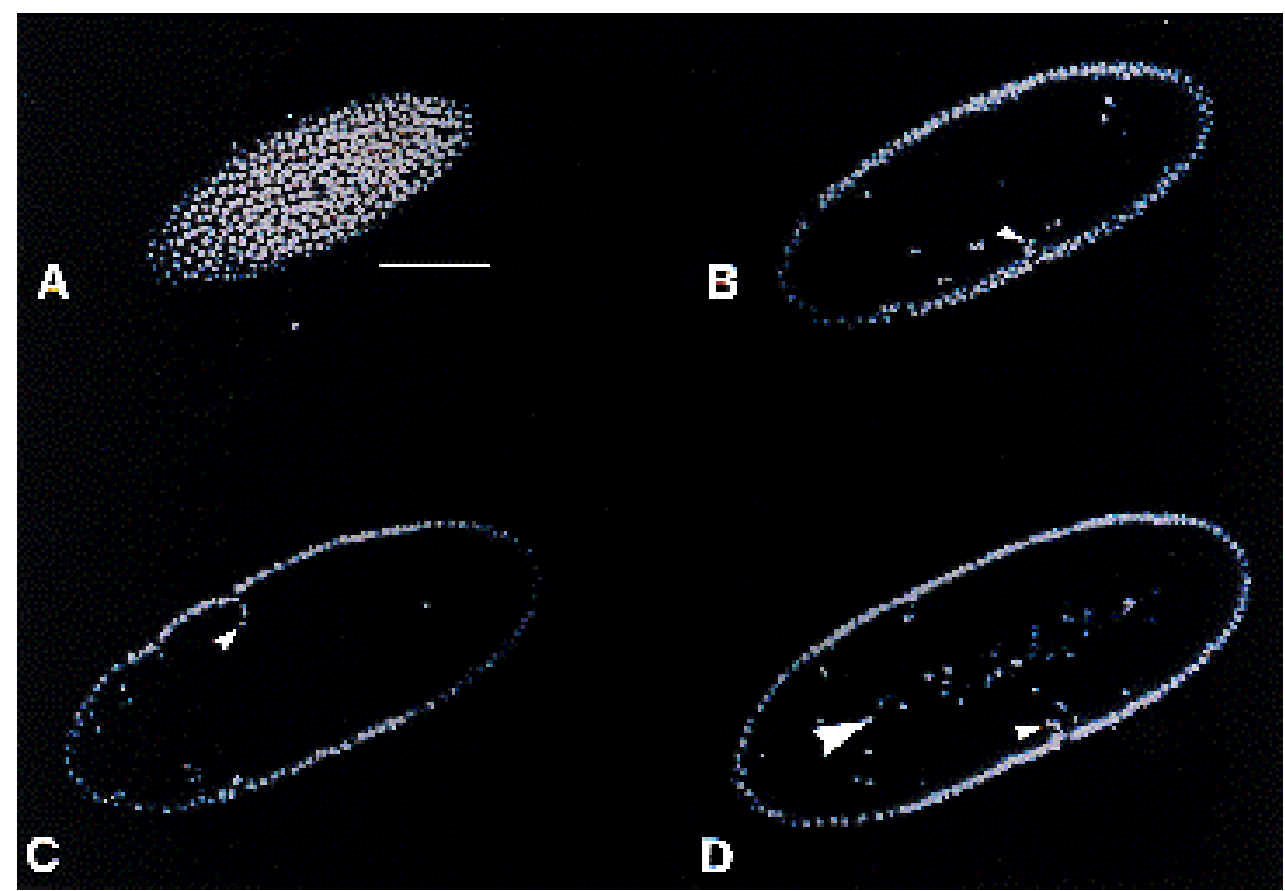

Fig. 4. Optical sections of a syncytial thr embryo. The embryo shown has been stained with propidium iodide to reveal DNA. The uppermost section is shown in (A) with sections at $5 \mu \mathrm{m}$ intervals in subsequent panels. The large arrowhead in (D) shows the yolk nuclei, which are found in the centre of the embryo. The small arrowheads in (B) and (C) indicate nuclei that have sunk from the cortical region where they are more normally found. Bar, 100 $\mu \mathrm{m}$.

carried out $\mathrm{P}$ element-mediated germ-line transformation experiments (Spradling and Rubin, 1982) utilising the DNA segments RX2 derived from the cosmid 15C2 (Fig. 1B). The RX2 construct restored viability to $t h r^{1 B}$ and $t h r^{S J B 22}$ homozygotes, indicating that no sequences necessary for wild-type $t h r^{+}$function lie outwith this region (Materials and Methods).

\section{Sequence analysis of thr}

The open reading frame in the DNA sequence of cDNA 2B1 revealed the clone to be incomplete, lacking $5^{\prime}$ sequences (see Materials and Methods). We therefore also determined the sequence of genomic DNA encoding the $\mathrm{N}$-terminal region of the $t h r$ protein. In addition we used a PCR-based approach to obtain the $5^{\prime}$ end of the $t h r$ tran-
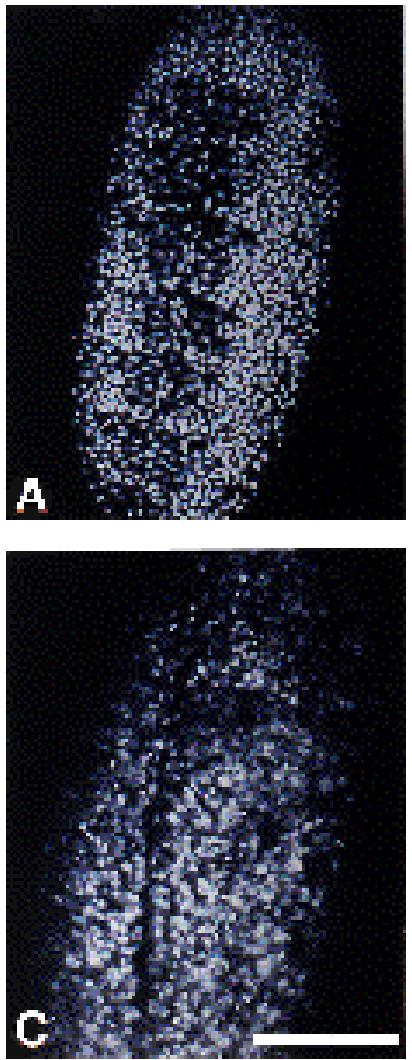
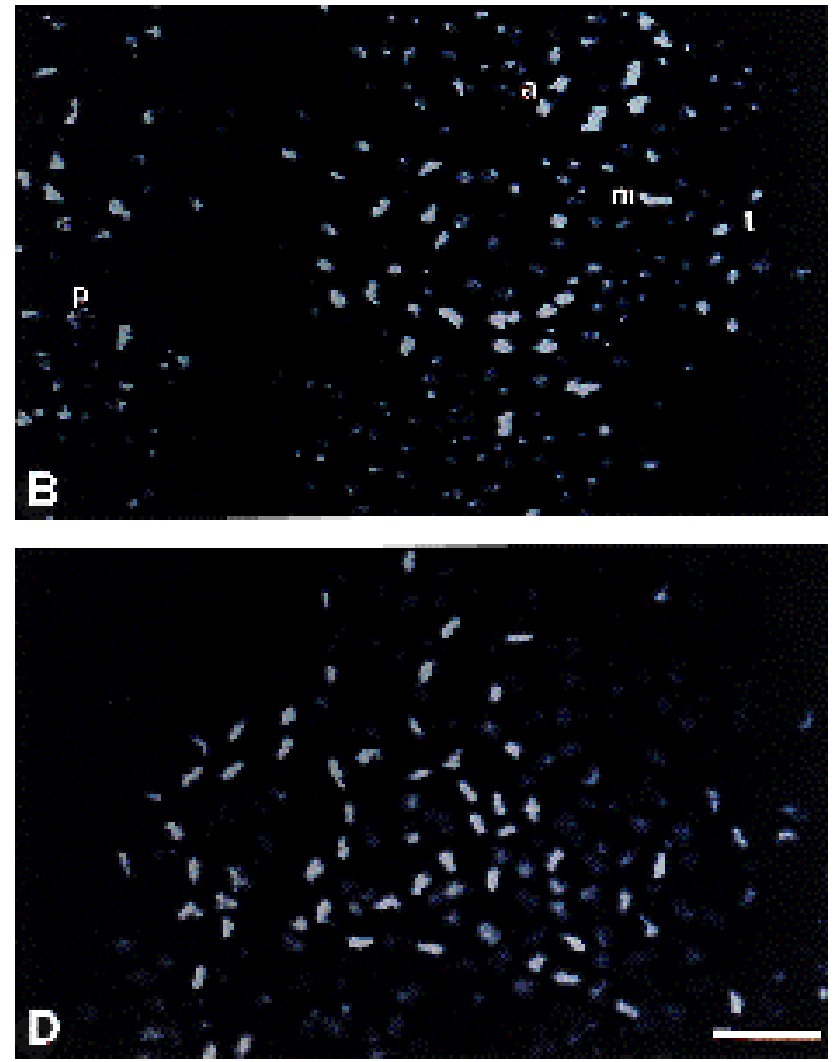

Fig. 5. Wild-type and thr embryos in cycle 15 . Wild-type (A and B) and $t h r(\mathrm{C}$ and $\mathrm{D})$ embryos are stained with propidium iodide. The wild-type arrangement of mitotic domains can be seen in the lowpower view of a wild-type embryo in (A). The higher magnification view in (B) reveals cells at all mitotic stages: prophase (p), metaphase (m), anaphase (a) and telophase (t). The $t h r$ embryo shows abnormally large domains of mitotic figures (C), which can be seen at higher power to contain mainly metaphase figures (D). Bars, $100 \mu \mathrm{m}(\mathrm{A}$ and $\mathrm{C})$ and $25 \mu \mathrm{m}(\mathrm{B}$ and D). 
script and have defined the start of the thr transcript to be $-722 \pm 5$ nucleotides upstream of the first in-frame start codon. The DNA sequence thus allows us to predict the size of the mRNA as 4432 nucleotides excluding the poly(A) tail. This is in excellent agreement with the results of northern analysis (data not shown). The transcripts have a similar distribution throughout early development to those of other genes essential for cell cycle functions. In situ hybridisation to embryos shows an abundant maternal contribution of $t h r$ mRNA to the syncytial embryo. These are excluded from newly formed cells during the interphase preceding mitosis 14 and are replaced in subsequent cycles by zygotic transcripts, present at particularly high levels in the developing central nervous system (data not shown).

The sequence of thr is presented as Fig. 2. The highlighted $\mathrm{M}$ at residue 1 is the first start codon in the reading frame but is not the best fit to the published consensus for translation start sites (Cavener and Ray, 1991). The second methionine (amino acid 43) best fits this consensus and is also a likely candidate for the actual translation start site. In this latter case the gene would encode a polypeptide of 1130 amino acids. The thr gene product bears little resemblance to any sequence reported in the databases. The only homology of possible significance detected is with the nuc2 gene product of Sshizosaccharomyces pombe. The match over a 26 amino acid sequence is as follows (|, identities; :, conservative matches):

$$
\begin{gathered}
\text { thr534NYASEAISAFSNFFDRADQEPLSANE559 } \\
:|:::||||::||||||:::| \\
\text { nuc2219SYSNSSISAFTKWFDRVDASELPGSE244 }
\end{gathered}
$$

This match occurs within the DNA binding domain of the nuc 2 gene product, a nuclear scaffold-like protein that appears to be required for chromosome disjunction (Hirano et al., 1988, 1990; Masuda et al., 1990.)

\section{Aberrant nuclear divisions occur at low frequency in syncytial thr embryos}

In order to search for mitotic abnormalities predicted by the cuticle phenotype, we first examined the organisation of chromatin in embryos lacking zygotic thr function (hereafter called thr embryos). The recessive thr mutation is maintained in a heterozygous stock with the $C y O$ balancer chromosome, which is marked with the E. coli lacZ gene. This enables homozygous thr embryos to be distinguished from their siblings by the lack of histochemically detectable $\beta$-galactosidase activity. thr embryos at successively later stages of development are shown in Fig. 3A to D. The normal distribution of nuclei in syncytial embryos (Fig. 3A) and newly cellularised embryos (Fig. 3B) suggests that mitosis takes place correctly in cycles 1-14. Mitotic abnormalities become strikingly evident in cellularised embryos during the second cell cycle to follow cellularisation (mitosis 15, Fig. 3C) and can be seen as large areas of cells delayed in mitosis (see below). The mutant embryo in (C) should be compared with the wild-type embryo at a comparable stage in (E). thr embryos subsequently develop large areas of cells that contain disorganised chromatin (Fig. 3D). The nuclei of many of these cells are considerably larger than those in wild-type embryos of comparable age (Fig. 3F).

A more careful inspection of thr embryos at syncytial blastoderm revealed that a small percentage of nuclei lose their association with the cortex during cycles 11 to 13 and appear to sink into the interior of the embryo. This can be seen in the four optical sections of a propidium iodidestained embryo presented as Fig. 4. The upper- and lower-
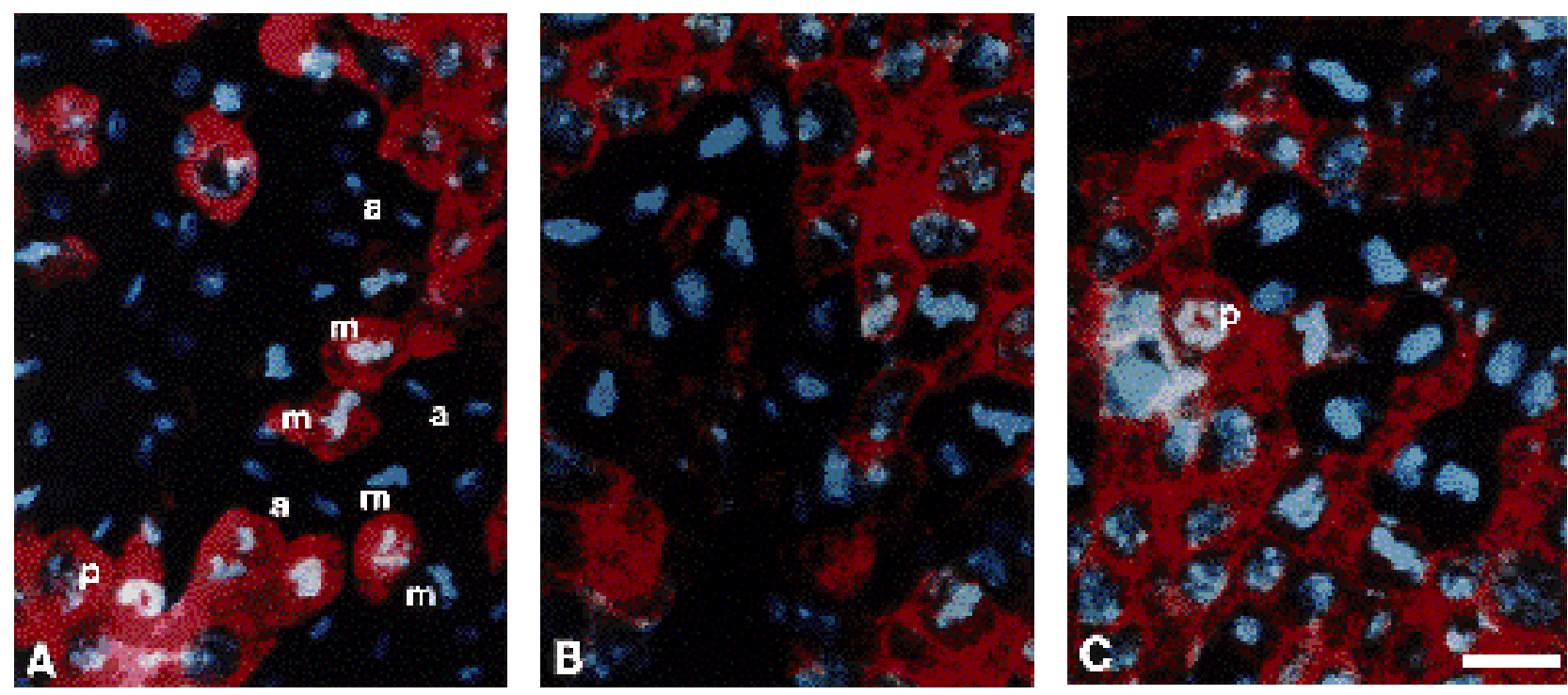

Fig. 6. Cyclin B degradation in mitotic domains of wild-type (A) and $t h r(B, C)$ embryos. Embryos have been stained with propidium iodide to reveal DNA (blue) and with the anti-cyclin B antibody described by Whitfield et al. (1990) to reveal cyclin B protein (red). In the wild-type embryo (A) cells can be seen at all mitotic stages: prophase (p), metaphase (m) and anaphase (a) are labelled. Cyclin B has been degraded in about half of the cells that have metaphase figures and in all the anaphase cells. In thr embryos (B and C) only prophase (p) and metaphase-like figures are visible. In embryos of this stage more than $95 \%$ of the metaphase cells lack cyclin B and there are no anaphase figures. Bar, $10 \mu \mathrm{m}$. 
most sections are through the layer of nuclei at the cortex of the syncytium (A) and the layer of yolk nuclei in the middle of the embryo (large arrowhead, D), respectively. Nuclei that have sunk below the cortex (small arrowheads, $\mathrm{B}$ and C) can be seen in the intermediate focal planes. We did not observe any such sinking nuclei in sibling embryos, which carried a $t h r^{+}$allele on the $C y O$ chromosome. This phenotype is similar to that previously described as a consequence of abnormal centrosome separation in the maternal effect mutant daughterless-abo-like (Sullivan et al., 1990), but considerably less severe. The low frequency with which this phenomenon occurs (less than $1 \%$ of nuclei appear to be affected in thr embryos) has meant that we have not been able to assess the nature of any mitotic defect that might lead to the phenomenon (see discussion).

\section{Delay in metaphase during cycle 15 of cellularised embryos}

We have examined the pattern of cycle 14 mitotic domains by following the behaviour of chromosomes stained using propidium iodide; spindle microtubules revealed by immunostaining; the nuclear antigen that relocalises from the nucleus to the centrosome during mitosis (Whitfield et al., 1988); and A- and B-type mitotic cyclins (data not shown). We conclude by each of these criteria that the order of entry into mitosis of each domain is as described by Foe (1989). We were unable to detect any mitotic abnormalities. We have also carried out pulse-labelling studies with BrdU that indicate that DNA synthesis occurs at the normal time, immediately following mitosis 14.

A comparison of the cycle 15 mitotic domains in wildtype and thr embryos is shown in Fig. 5. Whereas the wildtype domains contain cells at all stages of mitosis (A and $\mathrm{B})$, anaphase figures are not observed in thr embryos at this developmental stage, and instead mitotic domains contain predominantly metaphase figures (Fig. 5C and D). The mitotic domains of cycle 15 normally contain fewer cells than those of cycle 14. In thr embryos, however, the domains coalesce, giving rise to large patches of cells, all of which are at metaphase. We conclude that entry into mitosis 15 occurs normally but that cells are delayed in their mitotic progression after chromosome congression to the metaphase plate and before the separation of chromatids characteristic of anaphase.

\section{Some aspects of mitosis proceed in the absence of chromatid segregation}

In the cells of wild-type embryos, the mitotic cyclins accu-
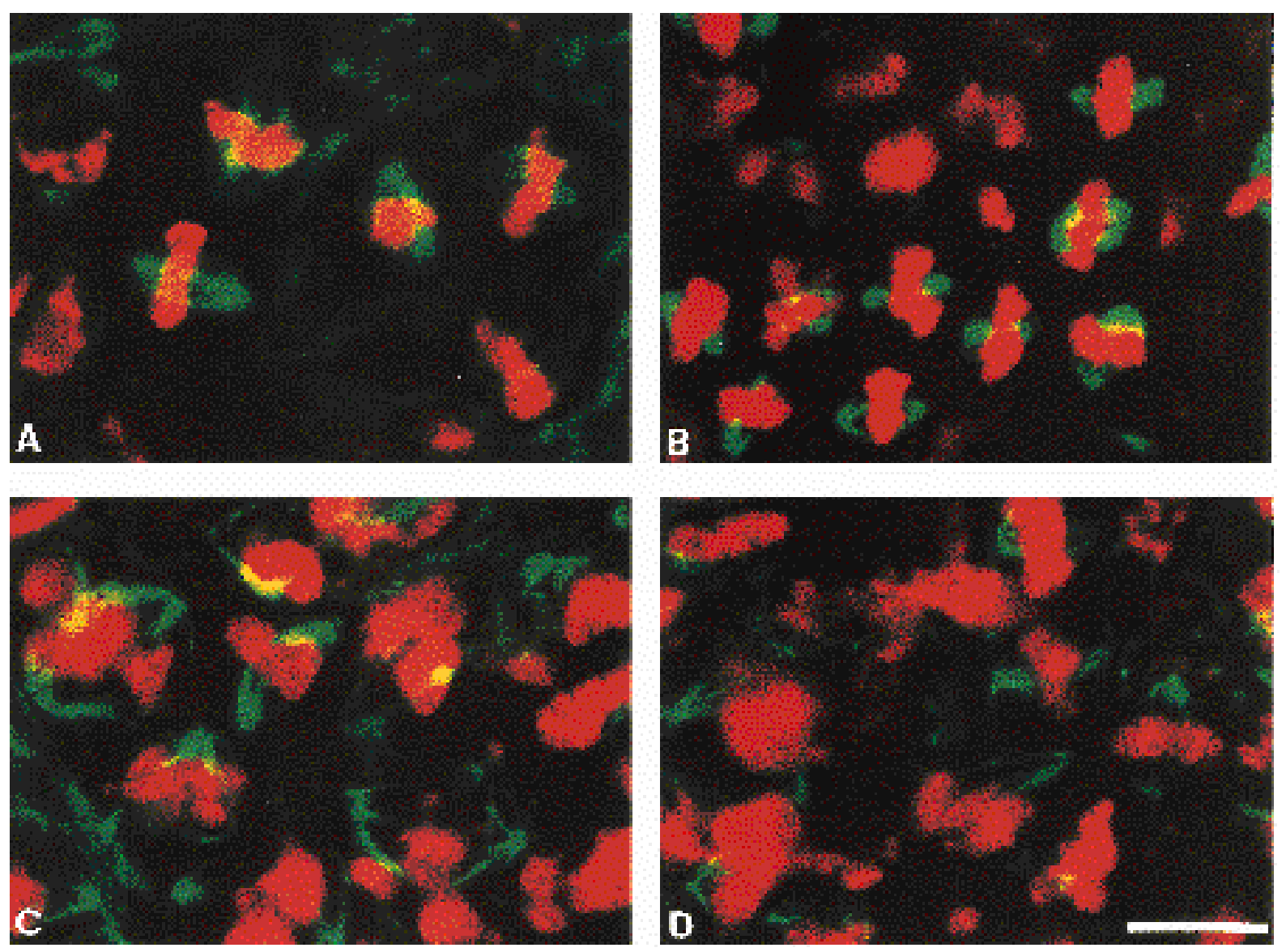

Fig. 7. Bipolar spindle structures in thr embryos. Embryos were stained for DNA (red) using propidium iodide, and for tubulin (green) using the YL1/2 monoclonal antibody (Kilmartin et al., 1982; Sera-lab). (A) to (C) show bipolar spindles associated with metaphase configurations of chromsomes. (C) and (D) are from a different embryo at a later stage in which the chromatin is decondensing. No chromatid separation is seen. Bar, $10 \mu \mathrm{m}$. 
mulate throughout interphase and into prophase (Lehner and O'Farrell, 1989, 1990; Whitfield et al., 1990). The cyclins are degraded at the metaphase-anaphase transition, cyclin A slightly ahead of cyclin B. Metaphase cells containing cyclin A are rare, whereas cyclin B is still present in about $50 \%$ of metaphase cells but has been completely degraded by the time that chromosome segregation occurs at anaphase (see Fig. 6A). In thr embryos cyclin B also accumulates in interphase and prophase cells but is not detectable in those cells delayed in metaphase (Fig. 6B and C). Cyclin A is also absent in these delayed cells (data not shown). Thus, it seems that the thr mutation delays metaphase in terms of chromatid separation, even though the metaphase-anaphase transition would appear to have taken place by the criterion of cyclin B degradation.

We wished to determine how this abnormal metaphaseanaphase transition might be resolved in thr cells and therefore carried out immunostaining to examine the organisation of the spindle microtubules (Fig. 7). We were able to detect bipolar mitotic spindles associated with chromatin in two different states. In the two mitotic domains shown in Fig. 7A and B, most cells have a metaphase configuration of chromosomes (red) associated with a bipolar mitotic spindle (green). This is the most common arrangement of thr spindles, and corresponds to the metaphase delays we have already described. Fig. 7C and D shows domains, from thr embryos, that appear to be at a later stage in these abnormal mitoses. The chromatin has remained at the position of the metaphase plate and is becoming decondensed as if at telophase.

\section{DISCUSSION}

A severe mitotic phenotype first becomes apparent in the thr embryos during cycle 15 . This suggests that the maternal provision of the $t h r$ gene product is sufficient to permit the embryo to develop more or less normally to this stage. This is reflected by the abundant supply of thr RNA detectable by in situ hybridisation in syncytial embryos (data not shown). Nevertheless, the finding of nuclei that from their position appear to be sinking from the cortex into the interior of the syncytial blastoderm embryo suggests that some defective mitoses might be occuring at this stage. We only detect this phenotype in embryos homozygous for $t h r$, and not in their siblings that will have received an equivalent, most likely reduced, contribution of the $t h r^{+}$gene product from their heterozygous mothers. It seems likely therefore that the levels of the $t h r^{+}$gene product in the syncytium of these embryos are near a critical threshold and must be supplemented by a zygotic contribution in order for all nuclei to divide normally. Such a contribution would come from expression of $t h r^{+}$in the unaffected embryos during syncytial blastoderm when low levels of expression are known to commence from several other genes. We have been unable to observe the nature of the mitotic defect at this developmental stage, and suggest that if the mutation results in a metaphase delay, as occurs in the later divisions, it would be difficult to detect in less than $1 \%$ of nuclei in a fixed preparation. The consequences of the aberrant division are more readily seen, however, in that the affected nuclei become abnormally located, and result in a small disruption of the monolayer of nuclei at the cortex around which unaffected nuclei continue to divide, thus leaving a 'footprint' that points to previously abnormal division. We are currently undertaking real time observations of mitoses in syncytial thr embryos to attempt to resolve this problem. Our failure to detect any aberrant mitoses in cycle 14 does not mean they do not occur. The asynchrony of divisions in mitotic domains accentuates the difficulty in detecting such events if they take place at low frequency.

The dramatic increase in abnormal mitoses during cycle 15 suggests that the maternal provision of $t h r^{+}$protein falls below a critical threshold at this time. In wild-type embryos, the majority of maternal $t h r$ transcripts are degraded at cellularisation, in common with many other mRNAs for cell cycle proteins (see Whitfield et al., 1989, for example). Although we do not as yet have antibodies to the $t h r^{+}$protein, and so cannot study its levels, we suspect that the maternal thr protein must persist beyond cellularisation, since the initial levels of zygotic transcription of the gene appear low. The zygotic transcripts subsequently increase considerably in the developing central nervous system (data not shown), a tissue that retains its mitotic potential throughout all of larval and pupal development.

Although all cells within a cycle 15 mitotic domain appear to be equally affected by thr mutations, the phenotype is not a cell cycle arrest, but rather a metaphase-like delay, followed by an apparent continuation of certain aspects of the cell cycle in the absence of chromatid segregation. This is perhaps surprising, since in the yeasts and in cultured mammalian cells, several dependency relationships have been described whereby the execution of a particular cell cycle event does not occur unless a previous step has been correctly completed (Hartwell and Weinert, 1989). Thus blocking the yeast cell cycle in $S$ phase prevents subsequent mitosis (Slater, 1973), and disruption of microtubules leads to a mitotic arrest that prevents the next $\mathrm{G}_{1}$ phase from taking place (e.g. see Huffaker et al., 1988). The existence of feedback mechanisms that regulate passage through these 'checkpoints' has been suggested by the discovery of mutants that fail to pause in response to DNA damage (Weinert and Hartwell, 1990), or fail to arrest in mitosis in response to loss of microtubule function ( $\mathrm{Li}$ and Murray, 1991; Hoyt et al., 1991). The checkpoint monitoring microtubule integrity would seem to be related to the need to assemble the mitotic apparatus correctly before proceeding with chromosome segregation. The drug-induced disruption of microtubules leads to a metaphase-like arrest in cells from a variety of organisms. Cyclin A within such arrested cells has undergone degradation, whereas cyclin B is maintained at high levels (Whitfield et al., 1989; Minshall et al., 1989). We assume that this arrest is at the checkpoint where correct assembly of the mitotic spindle is assessed. The delay produced by thr mutations, however, appears to be at a later stage in mitosis. Cells accumulate in metaphase with their chromosomes aligned on a normallooking spindle with both cyclins $\mathrm{A}$ and $\mathrm{B}$ having been degraded. It seems that the $t h r$ mutation prevents sister chromatid segregation, but that the resulting cell cycle delay occurs after the checkpoint for microtubule integrity. As the 
regulation of the segregation of chromatids into daughter cells at anaphase is crucial in cell division, the failure of cells to arrest at the known microtubule integrity checkpoint, before cyclin B degradation, in thr embryos was unexpected. This finding may therefore define a new checkpoint regulating chromatid segregation which is absent in $t h r$. Alternatively, $t h r^{+}$may have a purely mechanical function in mediating chromatid separation, and checkpoints, that detect the aberrant events of cycle 15, may only operate in the subsequent mitosis.

Mitosis normally consists of a linear progression of events. In the absence of $t h r^{+}$function, however, cells appear to accomplish only a subset of events in this linear progression: although cyclin B is degraded as normally happens at the metaphase-anaphase transition, the chromosomes maintain an appearance characteristic of metaphase and do not undergo any anaphase segregation. Telophase does occur in that the chromosomes subsequently decondense, and this is later followed by DNA replication. The linear execution of parts of the mitotic process in the absence of others in thr cells suggests the existence of parallel mitotic pathways that are normally coupled together. It is possible that thr functions as part of such a coupling mechanism, or that it is required to act in one of the pathways at the time at which coupling is achieved.

Such coupling processes may be related to the mitotic checkpoints, which appear to be introduced sequentially into the Drosophila cell cycle as development proceeds. Thus, in syncytial embryos (cycles 1-13), there appears to be no checkpoint coupling mitosis to the completion of Sphase (Raff and Glover, 1988). This dependence is only introduced following cellularisation in cycle 14, so that progression through cycles 14,15 and 16 appears to be regulated primarily at the $\mathrm{G}_{2}-\mathrm{M}$ transition by string, a Drosophila homologue of the fission yeast $c d c 25$ gene (Edgar and O'Farrell, 1989, 1990). A G 1 phase is not introduced until after cycle 16. Regulation in cell cycle progression at $G_{1}$ is known to occur in the central nervous system during larval development (Selleck et al., 1992). As thr is highly expressed in neural lineages, the cells of which go on to divide extensively after mitosis 16 , it will be of interest to examine the consequences of the thr mutations in such tissues at these and later developmental stages. Such an analysis would involve making mutant clones of thr cells, for example by mitotic recombination, in a heterozygous animal.

The finding of a small region of similarity with the fission yeast gene nuc2 is intriguing, as mutations in this gene block chromosome segregation and lead to metaphase arrest in fission yeast. However, we do not see any repeating amino acid domains in the thr protein, a characteristic structural feature of $n u c 2$. The nuc2 protein is present in the scaffold-like fraction of $S$. pombe cell extracts, and also contains a DNA binding domain. We are currently raising antibodies against the $t h r$ protein produced in $E$. coli in the hope that such reagents will help us study its sub-cellular localisation in Drosophila cells, and its interaction with other molecules.

We are extremely grateful for the assistance of Fiona Cullen who made sequencing almost bearable. We thank Christiane Nus-
slein-Volhard for pointing out to D.M.G. in 1984 that $t h r$ was likely to be a mitotic mutant. We also thank Alfonso MartinezArias for drawing our attention to the dysgenic thr alleles. We are especially grateful to Sarah Bray for providing the marked $\mathrm{CyO}$ $f t z-l a c Z$ balancer and for the previously unpublished $t h r^{S J B 22}$ allele. In the course of this work we became aware that the laboratories of Robert Saint and Christian Lehner were carrying out similar experiments. They have been extremely helpful in generously exchanging data prior to publication. Flies were obtained from the Tübingen and Bloomington stock centers. Finally we thank our colleagues in the lab. Their robust criticism is always enlightening. The work was supported by the Cancer Research Campaign and the Wellcome Trust. A.V.P. holds a Wellcome Trust studentship.

\section{REFERENCES}

Axton, J., Dombradi, V., Cohen, P. T. W. and Glover, D. M. (1990). One of the protein phosphatase isoenzymes in Drosophila is essential for mitosis. Cell 63, 33-46.

Brown, N. and Kafatos, F. (1988). Functional cDNA libraries from Drosophila embryos. J. Mol.Biol. 203, 425-437.

Carmena, M., González, C., Casal, J. and Ripoll, P. (1991). Dosage dependence of maternal contribution to somatic cell division in Drosophila melanogaster. Development 113, 1357-1364.

Cavener, D. R. and Ray, S. C. (1991). Eukaryotic start and stop sites. Nucl. Acids Res. 19, 3185-3192.

Edgar, B. A. and O'Farrell, P. H. (1989). Genetic control of cell division patterns in the Drosophila embryo. Cell 57, 177-187.

Edgar, B. A. and O'Farrell, P. H. (1990). The three postblastoderm cell cycles of Drosophila embryogenesis are regulated in $\mathrm{G}_{2}$ by string. Cell 62, 469-480.

Foe, V. (1989). Mitotic domains reveal early commitment of cells in Drosophila embryos. Development 107, 1-22.

Foe, V. and Alberts, B. (1983). Studies of nuclear and cytoplasmic behaviour during the five mitotic cycles that precede gastrulation in Drosophila embryogenesis. J. Cell Sci. 61, 30-85.

Freeman, M. and Glover, D. (1987). The gnu mutation of Drosophila causes inappropriate DNA synthesis in unfertilised and fertilised eggs. Genes Dev. 1, 924-930.

Freeman, M., Nusslein-Volhard, C. and Glover, D. M. (1986). Nuclear antigens follow different pathways into daughter nuclei during mitosis in early Drosophila embryos. J. Cell Sci. 82, 155-172.

Frischauf, A.-M., Lehrach, H., Poustka, A. and Murray, N. (1983). Lambda replacement vectors carrying polylinker sequences. J. Mol. Biol. 170, 827-842.

Gatti, M. and Baker, B. (1989). Genes controlling essential cell cycle functions in Drosophila melanogaster. Genes Dev. 3, 438-453.

González, C., Saunders, R. D. C., Casal, J., Molina, I., Carmena, M., Ripoll, P. and Glover, D. M. (1990). Mutations at the asp locus of Drosophila lead to multiple free centrosomes in syncytial embryos, but restrict centrosome duplication in larval neuroblasts. J. Cell Sci. 96, 605616.

Hartenstein, V. and Posakony, J. W. (1990). Sensillum development in the absence of cell division - the sensillum phenotype of the Drosophila mutant string. Dev. Biol. 138, 147-158.

Hartwell, L. H and Weinart, T. A. (1989). Checkpoints: Controls that ensure the order of cell cycle events. Science 246, 629-634

Henikoff, S. (1987). Unidirectional digestion with exonuclease III in DNA sequence analysis. Meth. Enzymol. 155, 156-180.

Hirano, T., Hiraoka, Y. and Yanagida, M. (1988). A temperaturesensitive mutation of the Schizosaccharomyces pombe gene nuc2 ${ }^{+}$that encodes a nuclear scaffold-like protein blocks spindle elongation in mitotic anaphase. J. Cell. Biol. 106, 1171-1183.

Hirano, T., Kinoshita, N., Morikawa, K. and Yanagida, M. (1990). Snap helix with knob and hole: essential repeats in $S$. pombe nuclear protein nuc $2^{+}$. Cell 60, 319-328.

Hoyt, M. A., Totis, L. and Roberts, B. T. (1991). S. cervisiae genes required for cell cycle arrest in response to loss of microtubule function. Cell 66, 507-517

Huffaker,T. C., Thomas, J. H. and Botstein, D. (1988). Diverse effects of 
$\beta$-tubulin mutations on microtubule formation and function J. Cell Biol. 106, 1997-2010.

Karess, R. E., Chang, X. J., Edwards, K. A., Kulkarni, S., Aguilera, I. and Kielhart, D. P. (1991). The regulatory light chain of nonmuscle myosin is encoded by spaghetti-squash, a gene required for cytokinesis in Drosophila. Cell 65, 1177-1189.

Kilmartin, J. V., Wright, B. and Milstein, C. (1982). Rat monoclonal antitubulin antibodies derived by using a new non-secreting rat cell line. $J$. Cell Biol. 93, 576-582.

Klemenz, R., Weber, U. and Gehring, W. J. (1987). The white gene as a marker in a new P element vector for gene transfer in Drosophila. Nucl. Acids Res. 15, 3947-3959.

Knoblich, J. and Lehner, C. (1993). Synergistic action of Drosophila cyclins $\mathrm{A}$ and $\mathrm{B}$ during the $\mathrm{G}_{2}$ transition. EMBO J. 12, 65-74.

Lehner, C. and O'Farrell, P. H. (1989). Expression and function of Drosophila cyclin A during embryonic cell cycle progression. Cell $\mathbf{5 6}$, 957-968.

Lehner, C. and O'Farrell, P. H. (1990). The roles of Drosophila cyclins A and $\mathrm{B}$ in mitotic control. Cell 61, 535-547.

Li, R. and Murray, A. W. (1991). Feedback control of mitosis in budding yeast. Cell 66, 519-531

Maldonado-Codina, G. and Glover, D. M. (1992). Cyclins A and B associate with chromatin and the polar regions of spindles, respectively, and do not undergo complete degradation at anaphase in syncytial Drosophila embryos. J. Cell Biol. 116, 967-976.

Masuda, H., Hirano, T., Yanagida, M. and Cande, W. Z. (1990). In vitro reactivation of spindle elongation in fission yeast nuc2 mutant cells. $J$. Cell Biol. 110, 417-425.

Minden, J. S., Agard, D. A., Sedat, J. W. and Alberts, B. A. (1989). Direct cell lineage analysis in Drosophila melanogaster by time-lapse three dimensional optical microscopy of living embryos. J. Cell Biol. 109, 505 516.

Minshall, J., Pines, J., Golsteyn, R., Standart, N., Mackie, S., Colman, A., Blow, J., Ruderman, J. V., Wu, M. and Hunt, T. (1989). The role of cyclin synthesis, modification and destruction in the control of cell division. J. Cell Sci. Suppl. 12, 77-97.

Nusslein-Volhard, C., Wieschaus, E. and Kluding, H. (1984). Mutations affecting the pattern of the larval cuticle in Drosophila melanogaster I Zygotic loci on the second chromosome. Roux's Arch. Dev. Biol. 193, 267-282.

O'Hare, K. and Rubin, G. M. (1983). Structures of P transposable elements and their sites of insertion and excision in the Drosophila melanogaster genome. Cell 34, 25-35.

Raff, J. W. and Glover, D. M. (1988). Nuclear and cytoplasmic mitotic cycles continue in Drosophila embryos in which DNA synthesis is inhibited with aphidicolin. J. Cell Biol.107, 2009-2019.

Robertson, H. M., Preston, C. R., Phillis, R. W., Johson-Schlitz, D., Benz, W. K. and Engels, W. R. (1988). A stable source of transposase in Drosophila melanogaster. Genetics 118, 461-470.

Sambrook, J., Fritsch, E. F. and Maniatis, T. (1989). Molecular Cloning: a Laboratory Manual, 2nd edn. CSH Press, Cold Spring Harbor Laboratory, New York.

Saunders, R. D. C., Glover, D. M., Ashburner, M., Sidén-Kiamos, I., Louis, C., Monastirioti, M., Savakis, C. and Kafatos, F. (1989). PCR amplification of DNA microdissected from a single polytene chromosome band: a comparison with conventional microcloning. Nucl. Acids Res 17, 9027-9037.

Selleck, S. B., González, C., Glover, D. M. and White, K. (1992). Regulation of the $\mathrm{G}_{1}-\mathrm{S}$ transition in post-embryonic neuronal precursors by axon ingrowth. Nature 355, 253-255.

Shamanski, F. L. and Orr-Weaver, T. L. (1991). The Drosophila plutonium and pan gu genes regulate entry into $\mathrm{S}$ phase at fertilisation. Cell 66, 1289-1300.

Sidén-Kiamos, I., Saunders, R. D. C., Spanos, L., Majerus, T., Treanear, J., Savakis, C., Louis, C., Glover, D. M., Ashburner, M. and Kafatos, F. C. (1990). Towards a physical map of the Drosophila melanogaster genome: mapping of cosmid clones within defined genomic divisions. Nucl. Acids Res. 18, 6261-6270.

Slater, M. L. (1973). Effect of reversible inhibition of deoxyribonucleotide acid synthesis on the yeast cell cycle. J. Bacteriol. 113, 263-270.

Spradling, A. C. and Rubin, G. M. (1982). Transposition of cloned P elements into Drosophila germ line chromosomes. Science 218, 347-362

Sullivan, W., Minden, J. S. and Alberts, B. A. (1990). daughterless-abolike a Drosophila maternal effect mutation that exhibits abnormal centrosome separaration during the late blastoderm divisions. Development 110, 311-323.

Szabad, J. and Bryant, P. J. (1982). Mode of action of discless mutations. Dev. Biol. 93, 240-256.

Tearle, R. and Nusslein-Volhard, C. (1988). Tubingen stock list. Dros. Inf. Service 66, 209-226.

Warn, R. N., Flegg, L. and Warn, L. (1987). An investigation of microtubule organisation and functions in living Drosophila embryos by injection of a fluorecently labelled antibody against tyrosinated tubulin. $J$. Cell Biol. 105, 1721-1730.

Weinart, T. A. and Hartwell, L. H. (1990). Characterisation of RAD9 of Saccharomyces cerevisiae and evidence that its function acts posttranslationally in cell cycle arrest after DNA damage. Mol. Cell Biol. 10, 6554-6564

Whitfield, W. G. F., González, C., Maldonado-Codina, G. and Glover, D. M. (1990). The A- and B- type cyclins of Drosophila are accumulated and destroyed in temporally distinct events that define separate phases of the $\mathrm{G}_{2}-\mathrm{M}$ transition. EMBO J. 9, 2563-2572.

Whitfield, W. G. F., González, C., Sanchez-Herrero, E. and Glover, D. M. (1989). Transcripts of one of two Drosophila cyclin genes become localised in pole cells during embryogenesis. Nature 338, 337-340.

Whitfield, W. G. F., Millar, S. E., Saumweber, H., Frasch, M. and Glover, D. M. (1988). Cloning of a gene encoding an antigen associated with the centrosome in Drosophila. J. Cell Sci. 89, 467-480.

(Received 10 May 1993 - Accepted 28 May 1993) 